EPJ Web of Conferences 52, 06001 (2013)

DOI: $10.1051 /$ epjconf/20135206001

(C) Owned by the authors, published by EDP Sciences, 2013

\title{
Results from the Pierre Auger Observatory
}

\author{
Lorenzo Perrone $^{1, \text { a }}$ for the Pierre Auger Collaboration ${ }^{2}$ \\ ${ }^{1}$ Università del Salento and INFN Lecce \\ ${ }^{2}$ Observatorio Pierre Auger, Av. San Martín Norte 304, 5613 Malargüe, Argentina \\ (Full author list: http://www.auger.org/archive/authors_2012_12.html)
}

\begin{abstract}
The Pierre Auger Observatory has been designed to investigate the origin and the nature of Ultra High Energy Cosmic Rays using a hybrid detection technique. It is located in the Province of Mendoza, Argentina, and consists of a surface array of about $3000 \mathrm{~km}^{2}$ overlooked by 27 air fluorescence telescopes grouped in four sites, which together provide a powerful instrument for air shower reconstruction. The combination of information from the surface array, measuring the lateral distributions of secondary particles at the ground, and the fluorescence telescopes, observing the longitudinal profile, enhances the reconstruction capability with respect to the individual detector components. Ultra High Energy Cosmic Rays offer also the unique chance of investigating particle interactions over an energy range well beyond the one covered by present and future ground-based particle accelerators. A review of selected results is presented with the emphasis given to the measurement of energy spectrum, arrival directions, chemical composition and the search for photons and neutrinos as primary particles.
\end{abstract}

\section{Introduction}

The Pierre Auger Observatory started collecting data in 2004 [1-3]. The Observatory uses hybrid measurements of air showers recorded by an array of 1660 water Cherenkov surface stations covering an area of $3000 \mathrm{~km}^{2}$, together with 24 air fluorescence telescopes that observe the development of air showers in the atmosphere above the array during dark nights.

An infill array [4] with half the grid size has been completed and is currently taking data with a threshold of about $3 \cdot 10^{17} \mathrm{eV}$. Moreover, three high-elevation telescopes (HEAT) [5] have begun operation and, together with the infill array in the FOV of the telescopes, will allow us to extend the hybrid measurements further down to $10^{17} \mathrm{eV}$ thus covering with full efficiency the region of the transition from galactic to extra-galactic cosmic rays. The deployment of buried muon detectors (AMIGA) [6] in the infill area is in progress and an extensive R\&D program for radio and microwave detection of UHE air showers is under way. The construction of the Auger Engineering Radio Array (AERA) has started [7] and several GHz-antennas are installed and taking data [8].

These extensions and new technologies may enhance the performance and capabilities of the Auger Observatory in Argentina and, in parallel, will explore their potential for a future much larger ground based observatory.

\section{The energy spectrum}

An accurate measurement of the cosmic ray flux above $10^{17} \mathrm{eV}$ is crucial because the features of the energy spectrum of ultra-high energy cosmic rays are intrinsically connected to the origin, nature and propagation of cosmic rays. At the highest energies, above $4 \times 10^{19} \mathrm{eV}$, a suppression of the flux has been observed by the HiRes experiment [9], the Pierre Auger Observatory [10-13] and the Telescope Array [14]. This suppression is compatible with the predicted Greisen-Zatsepin-Kuz'min (GZK) effect [15], even if other possibilities (e.g. limits in the maximum energy at the source) cannot be excluded. A break in the power law spectrum, named the "ankle", has also been reported around $10^{18.6} \mathrm{eV}$ [9-14], this feature being traditionally explained as the intersection of a steep Galactic spectral component with a flatter extragalactic one.

The energy spectrum above $2.5 \times 10^{18} \mathrm{eV}$ has been derived using data from the surface detector array $(\mathrm{SD})$ of the Pierre Auger Observatory [10]. The energy calibration of the SD-array is based on golden hybrid events, i.e. events that can be independently reconstructed from the surface array (SD) and the fluorescence telescopes (FD) [16].

SD data collected between 1 January 2004 and 31 December 2010 have been used for this analysis, which corresponds to an overall SD exposure of $20905 \mathrm{~km}^{2} \mathrm{sr}$ yr. The SD exposure is calculated by integrating the number of active detector stations of the surface array over time. Above $3 \times 10^{18} \mathrm{eV}$ the SD acceptance is saturated regardless of the primary mass and its uncertainty is about $3 \%$ [17]. The analysis is limited to events with zenith angle less than

\footnotetext{
a e-mail: lorenzo.perrone@le.infn.it
} 
$60^{\circ}$. A spectrum measurement using events with larger zenith angle is reported in [18].

The measurement of the energy spectrum has been extended to $10^{18} \mathrm{eV}$ [11-13] using FD events which also triggered at least one station of the surface detector array (hybrid events). Despite the limited number of events, due to the fluorescence detector on-time, the lower energy threshold and the good energy resolution of hybrid events allow us to measure the flux of cosmic rays with the standard array into the energy region where the transition between galactic and extragalactic cosmic rays is expected to occur.

The exposure of the hybrid mode of the Pierre Auger Observatory has been calculated using a time-dependent Monte Carlo simulation. The changing configurations of both fluorescence and surface detectors are taken into account for the determination of the on-time of the hybrid system. Within a time interval of $10 \mathrm{~min}$, the status and efficiency of all detector components of the Observatory, down to the level of the single PMTs of the fluorescence detector, are determined. Moreover, all atmospheric measurements [19] as well as monitoring information are considered and used as input for the simulation. A detailed description of the derivation of hybrid spectrum can be found in $[12,13]$.

The total systematic uncertainty on the FD energy scale is about $22 \%$, dominated by the uncertainty on the absolute fluorescence yield (14\%) [16].

The energy spectrum derived from hybrid data has been combined with the one obtained from surface detector data using a maximum likelihood method. Since the surface detector energy estimator is calibrated with hybrid events, the two spectra have the same systematic uncertainty in the energy scale. On the other hand, the normalisation uncertainties are independent. They are taken as $6 \%$ for the SD and $10 \%(6 \%)$ for the hybrid flux at $10^{18}$ $\mathrm{eV}\left(>10^{18} \mathrm{eV}\right)$. These normalisation uncertainties are used as additional constraints in the combination.

The characteristic features of the combined spectrum have been quantified with three power laws with free breaks between them (dashed line in Fig. 1) and with two power laws plus a smoothly changing function (solid line). The latter function is given by:

$$
J\left(E ; E>E_{\text {ankle }}\right) \propto E^{-\gamma_{2}} \frac{1}{1+\exp \left(\frac{\lg E-\lg E_{\frac{1}{2}}}{\lg W_{c}}\right)},
$$

where $E_{\frac{1}{2}}$ is the energy at which the flux has fallen to one half of the value of the power-law extrapolation and $W_{c}$ parametrizes the width of the transition region. The hypothesis that the power law above the ankle continues to highest energies with the spectral index $\gamma_{2}$ can be rejected with more than $20 \sigma$. The derived parameters with their statistical uncertainties are given in Table 1. Both, the ankle and suppression of the flux at higher energies are clearly visible.

The spectrum can be compared to astrophysical models and can be described by both a proton and heavydominated composition at the highest energies. Thus, measurements of the composition are needed to discriminate between various astrophysical models.

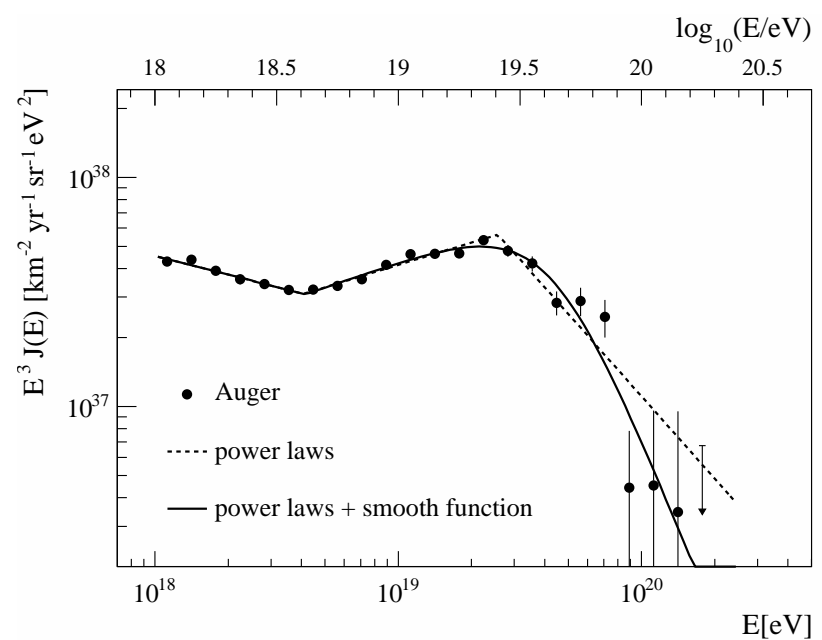

Figure 1. The combined Auger energy spectrum. A fit with three power law functions (dashed) and one with two power laws plus a smooth function (solid line) are superimposed. Only statistical uncertainties are shown. The systematic uncertainty on the energy scale is $22 \%$.

Table 1. Fit parameters with their statistical uncertainties

\begin{tabular}{l|r|r}
\hline parameter & broken power laws & $\begin{array}{r}\text { power laws } \\
\text { + smooth function }\end{array}$ \\
\hline$\gamma_{1}\left(E<E_{\text {ankle }}\right)$ & $3.27 \pm 0.02$ & $3.27 \pm 0.01$ \\
$\lg \left(E_{\text {ankle }} / \mathrm{eV}\right)$ & $18.61 \pm 0.01$ & $18.62 \pm 0.01$ \\
$\gamma_{2}\left(E>E_{\text {ankle }}\right)$ & $2.68 \pm 0.01$ & $2.63 \pm 0.02$ \\
$\lg \left(E_{\text {break }} / \mathrm{eV}\right)$ & $19.41 \pm 0.02$ & \\
$\gamma_{3}\left(E>E_{\text {break }}\right)$ & $4.2 \pm 0.1$ & \\
$\lg \left(E_{\frac{1}{2}} / \mathrm{eV}\right)$ & & $19.63 \pm 0.02$ \\
$\lg \left(W_{\mathrm{c}} / \mathrm{eV}\right)$ & & $0.15 \pm 0.02$ \\
$\chi^{2} / \mathrm{ndof}$ & $37.8 / 16=2.4$ & $33.7 / 16=2.1$ \\
\hline
\end{tabular}

\section{Mass Composition}

The measurement of the cosmic ray flux has to be complemented by an independent measurement of primary mass composition, with the goal of separating the different scenarios of origin and propagation of cosmic rays.

The composition can be obtained from measurements of various shower observables, primarily the atmospheric depth at which the shower reaches its maximum size, $X_{\max }$.

As the fluorescence telescopes can directly observe the shower longitudinal profile, hybrid events are used for this analysis. The position of $X_{\max }$ is derived from a fit to a Gaisser-Hillas function. Only events with a precise reconstruction of the geometry and of the longitudinal profile are selected. The $X_{\max }$ has to be observed in the FD field of view and quality cuts on the aerosol content and cloud coverage are applied. To avoid mass-dependent biases due to the limited field of view of the FD, the analysis is restricted to a geometrical volume that equalizes 
the selection efficiency over the major part of the parent $X_{\text {max }}$ distribution [20, 21].

Hybrid data collected between December 2004 and September 2010 have been used and 6744 events (with E $>10^{18} \mathrm{eV}$ ) fulfill the selection criteria. In Fig. $2\left\langle X_{\max }\right\rangle$ and its RMS are shown as a function of energy [21]. Both show a characteristic change at $\mathrm{E} \simeq 510^{18} \mathrm{eV}$ indicating an increasingly heavier composition when compared to air shower simulations. The $X_{\max }$ resolution is found to be about $20 \mathrm{~g} \mathrm{~cm}^{-2}$ [20] after detailed simulations of the detector and several cross-checks with events observed by two or more FD sites.

As mentioned in the previous section, the systematic uncertainty on the energy scale is about $22 \%$. The total systematic uncertainty on $\left\langle X_{\max }\right\rangle$ ranges between $10 \mathrm{~g} \mathrm{~cm}^{-2}$ at low energy and $13 \mathrm{~g} \mathrm{~cm}^{-2}$ at high energy. It includes contributions from the uncertainties in the calibration, the atmospheric data, the reconstruction and the event selection. The systematic uncertainty on $\operatorname{RMS}\left(X_{\max }\right)$ has been quoted as $5 \mathrm{~g} \mathrm{~cm}^{-2}$.

A detailed comparison of Auger results with other experiments, namely HiRes, Telescope Array and Yakutsk, has been shown in [22].

Although the SD cannot directly observe the $X_{\max }$, several observables (e.g. muon content, muon production depth, azimuthal asymmetry of the signal rise time) are related to the mass of the primary particle and to the shower development. The mass sensitive observables from SD can provide complementary information, with independent statistical uncertainties. The higher statistics allow us to extend this measurement to higher energies than possible with the FD. Details and a comparison between the resulting composition from SD and FD-based measurements are given in [21].

The position of the shower maximum is also influenced by the depth of the first interaction, i.e. by the crosssection of the primary particle with air. As a consequence, the interpretation of shower observables in terms of primary mass suffers from the lack of knowledge of interactions properties at the highest energies, propagating into air shower simulations. The next section will summarize the status of cross-section measurement carried out with the Pierre Auger Observatory.

\section{The proton-air cross-section}

Auger hybrid data provide a tool to investigate the matter interaction properties at energies well above the upper limits achievable with the accelerators nowadays available.

The tail of the $X_{\max }$ distribution is sensitive to the proton-air cross-section, a fact exploited in the pioneering work of the Fly's Eye Collaboration [23]. For this purpose, the shape of the distribution of the largest values of $X_{\max }$ is analyzed for a sample of hybrid events. The tail of $X_{\max }$ distribution that contains the $20 \%$ of deepest showers exhibits the expected exponential shape $\mathrm{dN} / \mathrm{dX}_{\max } \propto$ $\exp \left(\mathrm{X}_{\max } / \Lambda_{f}\right)$, see Fig. 3 . It is directly related to the $\mathrm{p}$-air cross-section via $\sigma_{p \text {-air }} \propto 1 / \Lambda_{f}$.
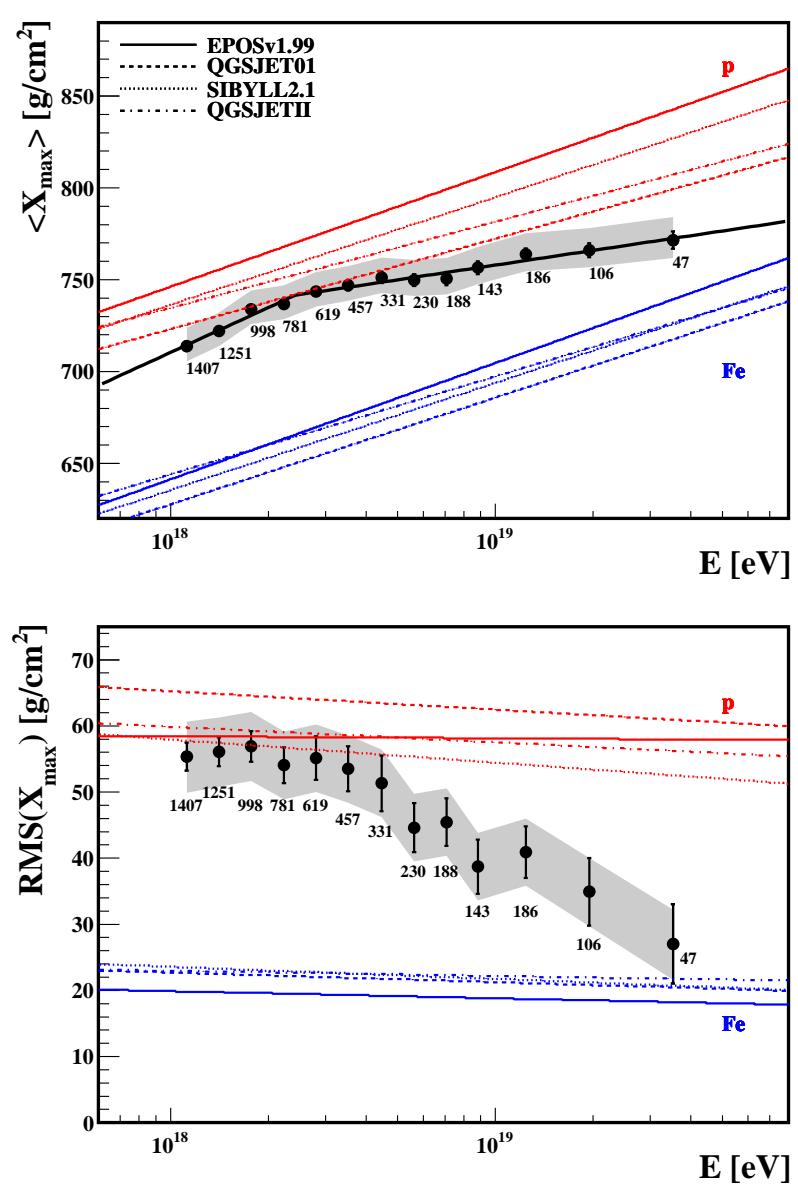

Figure 2. $\left\langle X_{\max }\right\rangle$ and $\operatorname{RMS}\left(X_{\max }\right)$ as a function of energy compared with air shower simulations using different hadronic interaction models [21].

In practice, to properly account for shower fluctuations and detector effects, the exponential tail is compared to Monte Carlo predictions. Any disagreement between data and predictions is then attributed to a modified value of the proton-air cross-section [25]. In this analysis, the energy interval is restricted between $10^{18}$ and $10^{18.5} \mathrm{eV}$ which corresponds to a center-of-mass energy in the nucleonnucleon system of $\sqrt{s}=57 \mathrm{TeV}$. This interval has been chosen because of high statistics in the data and because of the composition being compatible with a dominance of protons (see. Sec. 3).

A possible contamination of He primaries could mimic a larger cross-section (e.g. by $20 \mathrm{mb}$ for $20 \% \mathrm{He}$ contamination) while a photon contamination could reduce the cross-section by at most $10 \mathrm{mb}$.

Combining the results one finds

$$
\sigma_{p-a i r}=\left[505 \pm 22(\text { stat })_{-36}^{+28}(\text { syst })\right] \mathrm{mb}
$$

at a center-of-mass energy of $57 \pm 6 \mathrm{TeV}$. This result is shown in comparison to other data and models in Fig.4. The result favors a moderately slow rise of the crosssection towards higher energies, well in line with recent results from LHC [24]. 


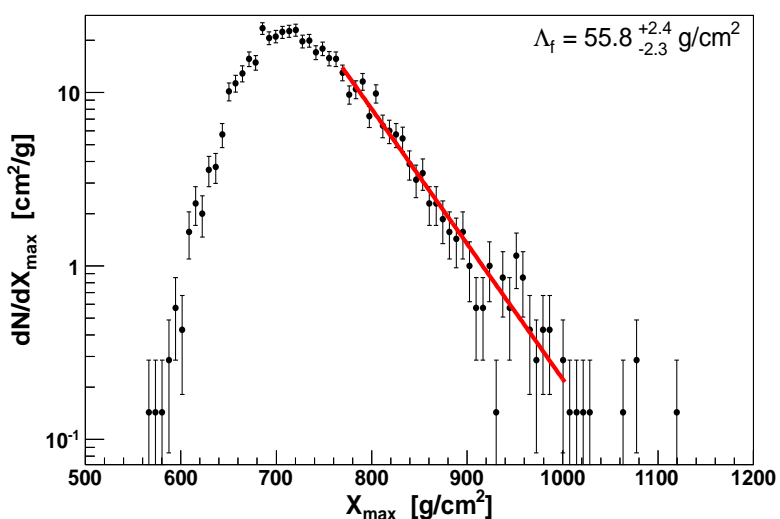

Figure 3. Unbinned likelihood fit to obtain $\Lambda_{f}$ (thick line).

A conversion of the derived $\sigma_{p-a i r}$ measurement into the more fundamental cross-section of proton-proton collisions using the Glauber framework is given in [25]. An exhaustive review of cross-section measurements with air shower experiments is presented by R. Ulrich at this Conference [26].

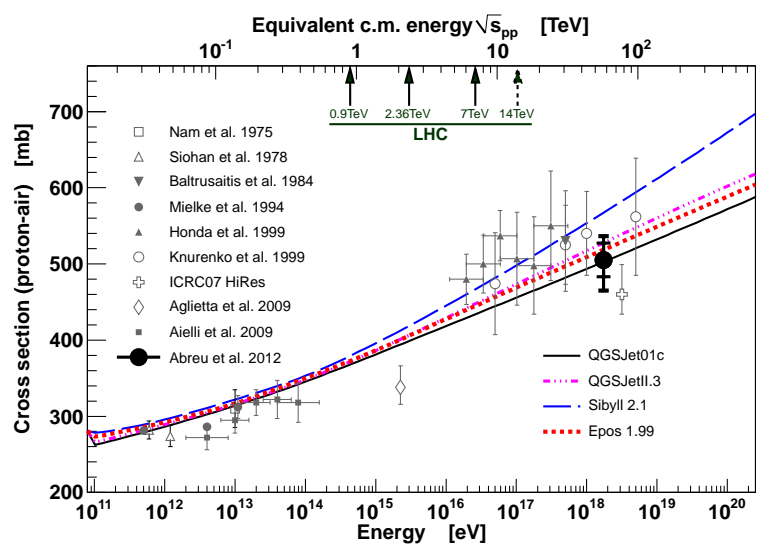

Figure 4. Proton-air cross-section compared to other measurements and model predictions (for references see [25]). The inner error bars are statistical only, while the outer include all systematic uncertainties for a helium fraction of $25 \%$ and $10 \mathrm{mb}$ photon systematics.

\section{Search for photons and neutrinos}

The search for ultra-high energy primary photons and neutrinos is motivated by several arguments. From an astrophysical point of view, the evidence of their existence would open a new window on the most extreme Universe. The detection of neutrinos and photons, as eventually produced by the decay of charged and neutral pions respectively, would also provide an independent proof of the GZK-effect.
Independently of a positive or negative result, their search will help constraining astrophysical scenarios for the origin and the propagation of UHECR, exotic models (i.e., Super Heavy Dark Matter, SHDM, topological defects, TD, Z-burst, etc., see [27] for a review) and provide hints of fundamental and new physics (e.g. Lorentz Invariance Violation [28]).

\subsection{Photons}

Distinctive characteristics of photon induced showers are a deeper shower maximum, $X_{\max }$, and a poorer muonic component. The delayed development of photon showers with respect to hadronic ones is due to the smaller multiplicity in electromagnetic interactions, and it is further delayed by the LPM effect [29] above $10 \mathrm{EeV}$. A dominating electromagnetic component is expected due to the lower cross-sections both for photo-nuclear interactions and muon pair production.

Upper limits on the absolute photon flux were derived by relating the number of photon-like events to the well known experimental exposure [30]. 95\% CL limits on the photon fraction of $2.0 \%, 5.1 \%$, and $31 \%$ above 10,20 , and $40 \mathrm{EeV}$, are derived (see Fig. 5, SD).

The key observable in searches for photon primaries with the fluorescence detector is the depth of shower maximum, $X_{\max }$. The difference between the average $X_{\max }$ value for showers induced by protons and photons at this energy is $\sim 200 \mathrm{~g} \mathrm{~cm}^{-2}$, which is large compared to the $X_{\max }$ resolution of the hybrid detector, $20 \mathrm{~g} \mathrm{~cm}^{-2}$ [20]. Observations in hybrid mode are possible also at energies below $10 \mathrm{EeV}$. By decreasing the energy threshold, the event statistics increases balancing, to some extent, the factor $\sim 10$ smaller duty cycle compared to observations with the ground array alone.

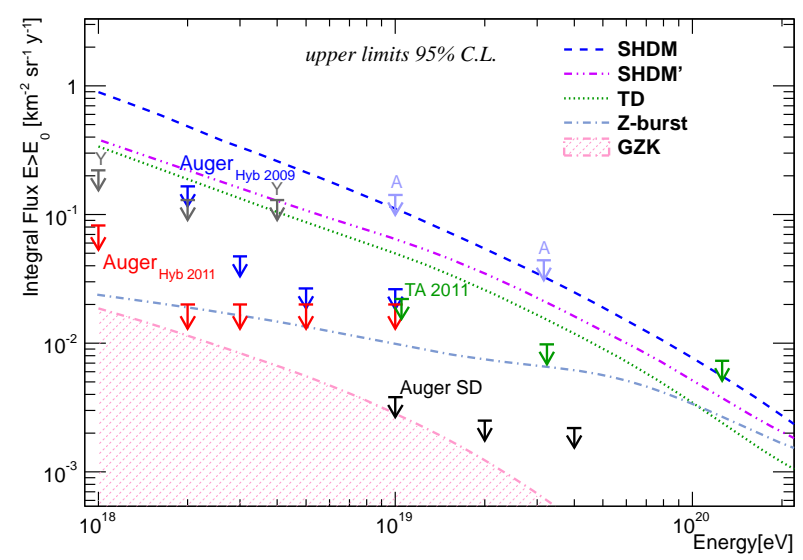

Figure 5. Upper limits on the integral photon flux [33] along with the previous ones by the Pierre Auger Observatory. See [32] for a complete list of references to experiments results and predictions. 
To improve the photon-hadron discrimination power the previous hybrid analyses [31], based on the FD $X_{\max }$ measurement only, are complemented in [33] with an SD observable combining the amplitude of the signal in the Cherenkov stations and the slope of the shower lateral distribution function. For the classification of photon candidates a Fisher analysis based on a large statistics Monte Carlo training sample is performed. Photon-like events are selected by applying an "a priori" cut at $50 \%$ of the photon selection efficiency. This provides a conservative result in the upper limit calculation by reducing the dependence on the hadronic interaction models and on the mass composition assumption. With this choice the expected nuclear contamination is about $1 \%$ in the lowest energy interval (between $10^{18}$ and $10^{18.5} \mathrm{eV}$ ) and it becomes smaller for increasing energies.

To carefully reproduce the operating conditions of the DAQ, time dependent simulations are performed according to the hybrid detector on-time. The actual configurations of the FD and SD and realistic atmospheric conditions are also taken into account.

Applying the method to data, 6, 0, 0, 0 and 0 photon candidates are found for energies above 1, 2, 3, 5 and $10 \mathrm{EeV}$. The observed number of photon candidates is consistent with the expectation from nuclear primaries, within the assumption of a mixed composition. Upper limits on the integral photon flux of $8.210^{-2} \mathrm{~km}^{-2} \mathrm{sr}^{-1} \mathrm{y}^{-1}$ above $1 \mathrm{EeV}$ and of $2.0 \cdot 10^{-2} \mathrm{~km}^{-2} \mathrm{sr}^{-1} \mathrm{y}^{-1}$ above 2, 3, 5 and $10 \mathrm{EeV}$ are derived [33].

Comparing the flux limits to the measured Auger spectrum [12, 13], upper bounds on the fraction of photons of about $0.4 \%, 0.5 \%, 1.0 \%, 2.6 \%$ and $8.9 \%$ are obtained for energies above 1, 2, 3, 5 and $10 \mathrm{EeV}$. The limits are shown in Fig. 5, (Hyb 2011) along with previous experimental results. The presented limits on the photon flux in UHECR favor astrophysical scenarios for the origin of the highest energy particles putting severe constraints on alternative non-acceleration models [27].

\subsection{Neutrinos}

Ultra high energy neutrinos of all flavours can induce extensive atmospheric showers that could be detected both by the Pierre Auger Observatory fluorescence and surface detectors [34-36].

Tau neutrinos can interact in the Earth crust producing, via charged current, a tau lepton which in turn can emerge and decay in the atmosphere, giving an Earth skimming upward-going (UG) event. If the decay occurs in flight over the detector, it may initiate a detectable air shower. Down-going (DG) events are showers induced by neutrinos of all flavours which interact in the atmosphere both via charged and neutral current.

The main challenge from the experimental point of view is to identify neutrino-induced showers in the large background of showers initiated by nuclear primaries. The observation of a significant electromagnetic component, at

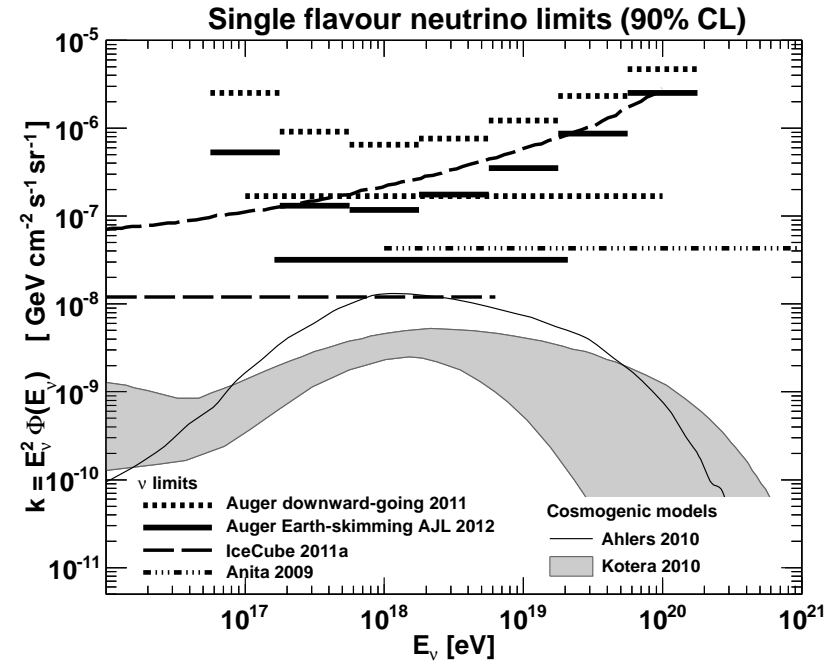

Figure 6. Differential and integrated upper limits at 90\% CL on the single flavour $\mathrm{E}_{v}^{-2}$ neutrino flux from the search for downward-going and Earth-skimming neutrinos at the Pierre Auger Observatory. Integrated upper limits are indicated by horizontal lines, with the corresponding differential limits being represented by segments of width 0.5 in $\log _{10} E_{\gamma}$. See Ref. [38] for a complete list of experiments results and predictions.

ground level, in events with very inclined arrival direction is the key to separate neutrino candidates from nuclear background.

Among events with zenith angles between $90^{\circ}$ and $95^{\circ}$ the selected candidates must exhibit elongated footprint at the ground, defined by a large length over width ratio, and mean propagation speed at ground close to the speed of light, for details see Refs. [37, 38]. The downward-going candidates were searched in a broader range of zenith angles, $75^{\circ}-90^{\circ}$.

The analysis methods were applied to data collected with the Auger surface detector, the exposure for UG (DG) neutrinos being equivalent to 3.5 (2) years of the full Auger SD. No candidate neutrino event was found in the collected data. Based on this, the corresponding limits on the diffuse flux of UHE for UG and DG neutrinos were derived. The total exposure of the Auger surface detector was computed with MC simulations, by folding the real time SD array aperture with the interaction probability and the method identification efficiency, for details see [39].

Several sources of systematic uncertainties were taken into account and their effect on the exposure evaluated. In particular the dominating systematic uncertainties are due to the lack of knowledge of the calculation of $\tau$ energy losses, the neutrino cross section at ultra-high energy.

Assuming a differential neutrino flux $\sim k \mathrm{E}^{-2}, 90 \%$ C.L. limits on neutrino flux were obtained, see Fig. 6. The limit on Earth skimming tau neutrinos, in the range 0.16-20.0 EeV, is $k<3.210^{-8} \mathrm{GeV} \mathrm{cm}^{-2} \mathrm{~s}^{-1} \mathrm{sr}^{-1}$ [38]. For down-going neutrinos in the range 0.1-100.0 EeV, $k<1.710^{-7} \mathrm{GeV} \mathrm{cm}^{-2} \mathrm{~s}^{-1} \mathrm{sr}^{-1}$ [37].

The sensitivity of the Pierre Auger Observatory to point-like sources of neutrinos with UHE has been obtained by evaluating the exposure as a function of source 
declination. The search for neutrinos was performed over a broad declination range, north of $\sim-65^{\circ}$ and south of $\sim 55^{\circ}$, and for neutrino energies between $10^{17} \mathrm{eV}$ and $10^{20} \mathrm{eV}$. Assuming a differential neutrino flux $\sim k_{P S} \mathrm{E}^{-2}$ from a point-like source, $90 \%$ confidence level upper limits for $k_{P S}$ at the level of $\sim 5 \cdot 10$ and $2.5 \cdot 10 \mathrm{GeV} \mathrm{cm}^{-2} \mathrm{~s}^{-1}$ have been obtained for Earth-skimming and downwardgoing neutrinos, respectively [38].

\section{Astrophysics}

One of the keys to understanding the nature of UHECRs is their arrival direction distribution over the sky. This distribution depends on the location of the UHECR sources, as well on the UHECR mass composition and large-scale magnetic fields, both Galactic and extragalactic. Despite significant efforts, none of these issues is well understood at present.

Observation of the suppression of the CR flux at the highest energies (see Sec. 2) and its interpretation in terms of the GZK-effect suggest that the sources of UHECRs are located within about $100 \mathrm{Mpc}$. At these scales the matter distribution in the Universe is inhomogeneous, and so must be the distribution of the UHECR sources. If propagation of UHECRs at these distances is quasi-rectilinear, anisotropies would be expected, showing variations at large angular scales and possibly point sources.

The Pierre Auger Collaboration in fact reported [40] directional correlations of UHECR at E $>5.510^{19} \mathrm{eV}$ with AGN from the Véron-Cetty-Véron catalog [41] within 75 $\mathrm{Mpc}$ on an angular scale of $3.1^{\circ}$ at the $99 \% \mathrm{CL}$.

The analysis including data up to June 2011 is shown in Fig.7. A total of 28 of 84 events show a correlation on a $3.1^{\circ}$-scale with a nearby AGN. The overall correlation strength thus decreased from $(62 \pm 10) \%$ initially to $(33 \pm 5) \%$. The chance probability of observing such a correlation from a random distribution remains below $1 \%$. The superimposed black symbols show in addition the averages of 10 independent consecutive events. The first bin is an upward fluctuation by about $3 \sigma$ from the mean of all events while the rest of the dataset does not show any peculiarity.

Evidently, more data is needed to draw a definite conclusion.

\section{Conclusions and Acknowledgements}

Selected results of the Pierre Auger Observatory have been presented. The Observatory will continue to collect data with unprecedented precision for several more years. An R\&D phase for studying possible upgrades of the Observatory has now started, mainly addressed to unveiling the issue of mass composition and anisotropies at the highest energies.

Finally, I wish to thank the organizers for the beautiful scientific and cultural environment around us in Berlin.

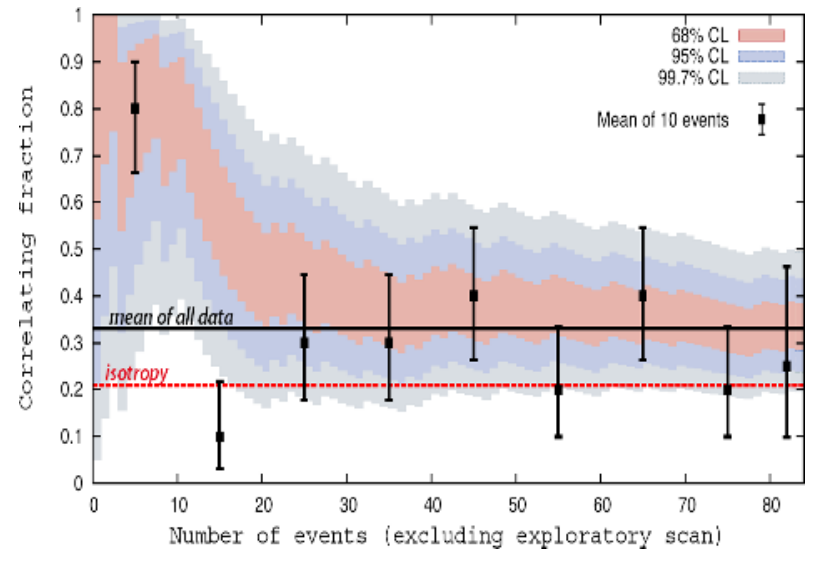

Figure 7. The most likely value of the degree of correlation $\mathrm{p}_{\text {data }}$ $=\mathrm{k} / \mathrm{N}$ is plotted as a function of the total number of time-ordered events (excluding those used for the exploratory scan). The 68\%, $95 \%$ and $99.7 \%$ confidence level intervals around the most likely value are shaded. The horizontal dashed line shows the isotropic value $\mathrm{p}_{\text {iso }}=0.21$ and the full line the current estimate of the signal $\mathrm{p}_{\text {data }}=0.33 \pm 0.05$. The black symbols show the correlation fractions bins of independent 10 consecutive events.

\section{References}

[1] The Pierre Auger Collaboration, Nucl. Instrum. Meth. A 523 (2004) 50.

[2] The Pierre Auger Collaboration, Nucl. Instrum. Meth. A 620 (2010) 227.

[3] I. Allekotte et al. for the Pierre Auger Collaboration, Nucl. Instrum. Meth. A 586 (2008) 409.

[4] I. Mariş for the Pierre Auger Collaboration, Proc. $32^{\text {nd }}$ ICRC, Beijing, China (2011), arXiv:1107.4809.

[5] H-J.Mathes, for the Pierre Auger Collaboration, Proc. $32^{\text {nd }}$ ICRC, Beijing, China (2011), arXiv:1107.4807.

[6] F. Sanchez, for the Pierre Auger Collaboration, Proc. $32^{\text {nd }}$ ICRC, Beijing, China (2011), arXiv:1107.4807.

[7] The Pierre Auger Collaboration, JINST 7 (2012) P11023.

[8] P. Facal for the Pierre Auger Collaboration, UHECR2012, CERN, February 13rd-17th, (2012).

[9] R. Abbasi et al., HiRes Collaboration, Phys. Rev. Lett. 100, (2008) 101101.

[10] The Pierre Auger Collaboration, Physical Review Letters 101, (2008) 061101.

[11] The Pierre Auger Collaboration, Physics Letters B 685, (2010) 239.

[12] F. Salamida, for the Pierre Auger Collaboration, Proc. 32 $2^{\text {nd }}$ ICRC, Beijing, China (2011), arXiv:1107.4809.

[13] M. Settimo for the Pierre Auger Collaboration, Eur. Phys. J. Plus 127 (2012) 87.

[14] Telescope Array Collaboration, submitted to PRL, arXiv:1205.5067v1.

[15] K. Greisen, Phys. Rev. Lett.16, (1966) 748. G. T. Zatsepin, V.A. Kuz'min, Pis'ma Zh. Eksp. Teor. Fiz., 4(3), (1966) 114. 
[16] R. Pesce for the Pierre Auger Collaboration, Proc. $32^{\text {nd }}$ ICRC, Beijing, China (2011)arXiv:1107.4809.

[17] The Pierre Auger Collaboration, Nucl. Instrum. Meth. A613 (2010) 29.

[18] H. Dembinski, for the Pierre Auger Collaboration, Proc. 32 $2^{\text {nd }}$ ICRC, Beijing, China (2011), arXiv:1107.4809.

[19] K. Louedec, for the Pierre Auger Collaboration, Proc. 32 ${ }^{\text {nd }}$ ICRC, Beijing, China (2011), arXiv:1107.4806.

[20] The Pierre Auger Collaboration, Phys. Rev. Lett. 104 (2010), 091101.

[21] P. Facal, for the Pierre Auger Collaboration, Proc. $32^{\text {nd }}$ ICRC, Beijing, China (2011), arXiv:1107.4804.

[22] E. Barcikowski , J. Bellido, J. Belz, Y. Egorov, S. Knurenko, V. de Souza, Y. Tsunesada, and M. Unger for the HiRes, Pierre Auger, Telescope Array and Yakutsk Collaborations, UHECR-2012, CERN February 13rd-17th, (2012).

[23] R. Ellsworth et al., Phys. Rev. D 26, (1982) 336, R. Baltrusaitis et al., Phys. Rev. Lett. 52, (1984) 1380.

[24] TOTEM Collaboration, Europhys. Lett. 96, (2011) 21002; ATLAS Collaboration, Nature Commun. 2 (2011) 463; CMS Collaboration, arXiv:1205.3142v1.

[25] The Pierre Auger Collaboration, Phys. Rev. Lett. 109 (2012) 062002.

[26] R. Ulrich at this Conference.

[27] P. Bhattacharjee, G. Sigl, Phys. Rep. 327, (2000) 109.
[28] M. Galaverni and G. Sigl, Phys. Rev. Lett. 100, (2008) 021102.

[29] L.D. Landau, I.Ya. Pomeranchuk, Dokl. Akad. Nauk SSSR 92,(1953) 535 and 735, A.B. Migdal, Phys. Rev. 103, (1956) 1811.

[30] The Pierre Auger Collaboration, Astropart. Phys. 29, (2008) 243.

[31] The Pierre Auger Collaboration, Astropart. Phys. 27, (2007) 155, Astropart. Phys. 31, (2009) 399.

[32] J. Alvarez-Muniz and M. Risse for the Pierre Auger Coll., G. I. Rubtsov and B. T. Stokes for the Telescope Array Coll., UHECR-2012, CERN, February 13rd-17th, 2012.

[33] M. Settimo for the Pierre Auger Collaboration, Proc. $32^{\text {nd }}$ ICRC, Beijing, China (2011)arXiv:1107.4805.

[34] K. S. Capelle et al., Astropart. Phys. 8 (1998) 321.

[35] X. Bertou et al., Astropart. Phys. 17 (2002) 133.

[36] C. Aramo et al., Astropart. Phys. 23 (2005) 65.

[37] The Pierre Auger Collaboration, Phys. Rev. D 84, (2011) 122005.

[38] The Pierre Auger Collaboration, Astrophys. J. Lett L4, (2012) 755.

[39] The Pierre Auger Collaboration, Phys. Rev. D 79, (2009) 102001.

[40] The Pierre Auger Collaboration, Science 318 (2007) 938; Astropart. Phys., 29 (2008) 188; Astropart. Phys., 34 (2010) 314.

[41] M.-P. Véron-Cetty, P. Véron, Astron. Astrophys., 445 (2006) 773. 